\title{
The exact solution of the problem on the forced oscillations of an elastic wedge shaped thick plate
}

\author{
N. Vaysfel'd
}

\begin{abstract}
A finite elastic wedge shaped thick plate is considered. One of the faces is based on a rigid base, other is exposed to a dynamic oscillating loading through an absolutely rigid plate. On the side faces the conditions of the smooth contact are given, at the plate's end stress are equal zero. The solving is based on a special linear transformation of Lame's equations and an applying of the matrix integral transformation method. The proposed approach leads to a one-dimensional vector boundary value problem for which an exact solution is constructed. The analysis of the eigen frequencies' distribution and resonance's frequencies are done.
\end{abstract}

Keywords-wedge shaped thick plate, integral transformation, vector boundary problem, exact solution

\section{Introduction}

The studies of oscillatory processes held today detail and extensively [1-6], due as to the wide practical applications of the research's results in an engineering, so and to a development of the mathematical solving methods for the problems of the deformable bodies' dynamics. It should be noted that the problem for the wedge-shaped bodies in view of the complexity of its geometry is studied much less than the problems for the bodies of the cylindrical and spherical shapes, the circular disks and plates. In a static statement the problems for the wedge plates are considered in $[7,8]$ a dynamic effect on the wedge-shaped plate was studied in [911], where the authors used the approximate methods for solving of the boundary value problems. The analytical solving method, which allow to obtain an exact solution of the three-dimensional problem of the wedge plate's free vibrations with some special boundary conditions on its surface, was proposed in [12].

One of the important directions in the studying of the oscillatory processes is a determination the elastic bodies' eigenfrequencies. These investigations are well known for

Nataly Vaysfel'd

Odessa Mechnikov University

Ukraine the bodies of the canonical shapes, the finite elastic cylinders, the disks and the rectangle [11]. The studying of the eigenfrequencies for an wedge shaped plate apparently previously was not conducted. Also useful are the investigations of an edge resonance phenomenon, by which one means the eigen oscillations with the significant localized zones of an increased intensity of the dynamic stress state around the certain parts of the boundary surfaces. This phenomenon was studied in detail and comprehensively for the isotropic and transversely isotropic bodies of the canonical forms, but the studies for the elastic wedge shaped bodies in the literature are not marked.

In the proposed work an exact solution of the dynamic problem for a finite wedge plate is constructed. The investigation of the eigenfrequencies' spectrum is conducted. The edge resonance's frequencies are detected.

\section{The Problem's Statement and its Reducing to the one- dimensional vector boundary Problem}

The elastic wedge shaped thick plate $0 \leq \mathrm{r} \leq \mathrm{a}, 0 \leq \varphi \leq \omega, 0 \leq \mathrm{z} \leq \mathrm{h}$ is considered. A plate's face $\mathrm{z}=\mathrm{h}$ (the lower bound) is considered to be based on a rigid smooth base

$u_{z}(r, \varphi, h, t)=0, \quad \tau_{z r}(r, \varphi, h, t)=0, \quad \tau_{z \varphi}(r, \varphi, h, t)=0$

In the smooth contact with the face $\mathrm{z}=0$ is absolutely rigid plate of the known mass $\mathrm{m}$, which has the same form as the upper face of the plate. The concentrated force $\mathrm{P}(\mathrm{t})$ by the law $P \cos \varpi t$ ( $\varpi$ is the load's frequency) is applied to the rigid plate through the line $0 \leq \mathrm{r} \leq \mathrm{a}, \varphi=\omega / 2$ at the distance 1 from the vertex of the wedge. Under this force's influence the points of the upper bound are shifted at the value $\delta+\operatorname{Arcos} \varphi$, and the conditions on the face $\mathrm{z}=0$ can be written as

$u_{z}(r, \varphi, 0, t)=(\delta+A r \cos \varphi) \cos \tilde{\omega} t, \tau \tau_{z r}(r, \varphi, 0, t)=0, \tau_{z \varphi}(r, \varphi, 0, t)=0$

The unknown constants $\delta, \mathrm{A}$ are determined from the rigid plate movements' equations, which on the basis of the D’Alamber's principal are written as

$$
\begin{gathered}
-P(t)+\int_{-\pi}^{\pi} \int_{0}^{a} \sigma_{z}(r, \varphi, h, t) r d r d \varphi-\left.m \frac{\partial^{2} u_{z}}{\partial t^{2}}\right|_{z=h}=0 \\
-l P(t)+\int_{-\pi}^{\pi} \int_{0}^{a} \sigma_{z}(r, \varphi, h, t) r^{2} \cos \varphi d r d \varphi-\left.\frac{2 m a \sin ^{2} \omega}{3 \omega} \frac{\partial^{2} u_{z}}{\partial t^{2}}\right|_{z=h}=0
\end{gathered}
$$


The conditions of the smooth contact are fulfilled at the faces $\varphi=0, \varphi=\omega$

$$
\left.u_{\varphi}(r, \varphi, z, t)\right|_{\varphi=0, \omega}=0, \tau_{\varphi z}(r, \varphi, z, t)=\left.\right|_{\varphi=0, \omega}=0,\left.\tau_{\varphi r}(r, \varphi, z, t)\right|_{\varphi=0, \omega}=0
$$

On the face $r=a$ the conditions of the elasticity's first fundamental problem are assumed

$$
\sigma_{r}(a, \varphi, z, t)=0, \quad \tau_{r \varphi}(a, \varphi, z, t)=0, \quad \tau_{r z}(a, \varphi, z, t)=0
$$

It is required to find the solution of the problem that satisfies to all boundary conditions and the movements' equations for the displacements.

\section{The Problem's Reducing to the one-dimensional vector boundary Problem}

One must apply the Fourier's transformations with regard to the variable $\varphi$ to the movements' equations and the boundary conditions by the scheme

$$
\left[\begin{array}{c}
u_{n}(r, z) \\
w_{n}(v, z)
\end{array}\right]=\int_{0}^{\omega} \cos \alpha_{n} \varphi\left[\begin{array}{l}
u(r, \varphi, z) \\
w(r, \varphi, z)
\end{array}\right] d \varphi ; \quad n=0,1,2, \ldots
$$

$v_{n}(r, z)=\int_{0}^{\omega} \sin \alpha_{n} \varphi v(r, z, \varphi) d \varphi ; \quad n=1,2,3, \ldots ; \quad \alpha_{n}=\frac{\pi n}{\omega}$

Here and further is assumed that

$$
\begin{gathered}
u_{r}(r, \varphi, z, t)=u(r, \varphi, z) \cos \tilde{\omega} t, \\
u_{\varphi}(r, \varphi, z)=v(r, \varphi, z) \cos \tilde{\omega} t, \\
u_{z}(r, \varphi, z, t)=w(r, \varphi, z) \cos \tilde{\omega} t
\end{gathered}
$$

During the procedure's executing the boundary conditions at the faces $\varphi=0, \varphi=\omega$ were satisfied. To the transformed equations and the boundary conditions at the bottom and upper faces the Fourier's transformations with regard to the variable $\mathrm{z}$ were applied

$$
\begin{gathered}
{\left[\begin{array}{c}
u_{n k}(r) \\
v_{n k}(r)
\end{array}\right]=\int_{0}^{h} \cos \beta_{k} z\left[\begin{array}{l}
u_{n}(r, z) \\
v_{n}(r, z)
\end{array}\right] d z ; \quad \beta_{k}=\frac{\pi k}{h}, \quad k=0,1,2, \ldots} \\
w_{n k}(r)=\int_{0}^{h} \sin \beta_{k} z w_{n}(r, z) d z, \quad k=1,2,3, \ldots
\end{gathered}
$$

\section{A. The Solving of the one-dimensional vector boundary Problem for the Case $n=0, k \geq 1$}

In this case the transformed movement's equations system is separated on the two equations, which with the corresponded boundary conditions are written in the form of the vector boundary problem

$$
\begin{aligned}
& \mathbf{L}_{0 k}\left(\mathbf{y}_{0 k}(r)\right)=\mathbf{f}_{0 k}(r), 0<r<a \\
& \mathbf{U}_{0 k}\left(\mathbf{y}_{0 k}(a)\right)=\mathbf{i}, i=0,1
\end{aligned}
$$

where

$$
\begin{gathered}
\mathbf{L}_{0, k}=\left(\begin{array}{cc}
D_{r}-r^{-2}-\beta_{k}^{2} \mu_{*}^{-1}+q^{2} \mu_{*}^{-1} & \mu_{0} \mu_{*}^{-1} \beta_{k} \partial_{r} \\
-\mu_{0} \beta_{k}\left(\frac{\partial}{\partial r}+\frac{1}{r}\right) & D_{r}-\beta_{k}^{2} \mu_{*}+q^{2}
\end{array}\right) \\
\mathbf{U}_{0, k}=\left(\begin{array}{cc}
\bar{\mu} a^{-1}+\partial_{r} & \bar{\mu} \beta \\
-\beta & 1
\end{array}\right), D_{r}=r^{-1}\left(r f^{\prime}\right)^{\prime}, \partial_{r}=f^{\prime}, \\
\mathbf{y}_{0 k}(r)=\left(\begin{array}{c}
u_{0 k}(r) \\
w_{0 k}(r)
\end{array}\right), \quad \mathbf{f}_{0 k}(r)=\left(\begin{array}{c}
\bar{\mu} \delta_{0}^{\prime}(r) \\
-\mu_{*} \beta_{k} \delta_{0}(r)
\end{array}\right) \\
\mathbf{i}(r)=\left(\begin{array}{c}
\bar{\mu} \delta_{0}(r) \\
0
\end{array}\right) .
\end{gathered}
$$

The notifications are taken here and further

$\delta_{n}(r)=2(-1)^{n} A r \sin \omega, \quad q^{2}=\frac{\tilde{\omega}^{2} \rho}{G}=\tilde{\omega}^{2} c^{2}$

$\mu_{*}=1+\mu_{0}, \bar{\mu}=\left(\mu_{0}-1\right) \mu_{*}^{-1}$

$\mu_{0}=(1-2 v)^{-1}, \mathrm{G}, \rho, v-$ are a shear modulus, a density and a Poisson's ratio correspondently, a stroke above a letter denotes the derivative with respect to the first variable.

For the solution's construction one must solve the matrix homogenous equation $L_{0 k}(\mathbf{Y}(r))=0,0<r<a_{[13,14] \text {. }}$ With this aim the matrix $\mathbf{H}_{\mathbf{0 k}}(\mathbf{r}, \mathbf{s})$ is found. The main property is the executing of the equality

$$
L_{0 k}\left(\mathbf{H}_{0 k}(r, s)\right)=-\mathbf{H}_{0 k}(r, s) \mathbf{M}_{0 k}(r, s), 0<r<a
$$

One can sure that matrix

$$
\mathbf{H}_{0 k}(r, s)=\left(\begin{array}{cc}
J_{1}(r s) & 0 \\
0 & J_{0}(r s)
\end{array}\right)
$$

satisfies to this demand ( the regularity in zero is guaranteed). The solution of the obtained matrix equation is found with the help of the residual's calculations with the formulas, which is proposed in [13]. After it the solution of the homogenous matrix equation is obtained 


$$
\begin{aligned}
& \mathbf{Y}(r)= \\
& \left\{\begin{array}{lc}
\left(\begin{array}{cc}
\frac{(-v+1) \beta^{2} J_{1}\left(\sqrt{q^{2}-\beta^{2}} r\right)}{\sqrt{q^{2}-\beta^{2}}} & \beta J_{1}\left(\sqrt{q^{2}-\beta^{2}} r\right) \\
\beta(v-1) J_{0}\left(\sqrt{q^{2}-\beta^{2}} r\right) & -\sqrt{q^{2}-\beta^{2}} J_{0}\left(\sqrt{q^{2}-\beta^{2}} r\right)
\end{array}\right) \\
\left(\begin{array}{cc}
\frac{(-v+1) \beta^{2} I_{1}\left(\sqrt{q^{2}-\beta^{2}} r\right)}{\sqrt{q^{2}-\beta^{2}}} & \beta i I_{1}\left(\sqrt{q^{2}-\beta^{2}} r\right) \\
\beta(v-1) I_{0}\left(\sqrt{q^{2}-\beta^{2}} r\right) & -i \sqrt{q^{2}-\beta^{2}} I_{0}\left(\sqrt{q^{2}-\beta^{2}} r\right)
\end{array}\right)
\end{array}\right.
\end{aligned}
$$

for two cases $q<\beta$ and $q>\beta$ correspondently. As it seen, the second column in the case $\mathrm{q}<\beta$ takes pure image values, that is why for the providing of the displacements' value realness at this case (with regard of the known correspondences for the Bessel's functions $\left.J_{0}(i z)=I_{0}(z), J_{1}(i z)=i I_{1}(z) \quad[15]\right)$, the solution of the initial problem is constructed in the form

$$
\begin{aligned}
& u_{0 k}(r)=C_{1} Y_{11}(r)+C_{2} Y_{12}(r), v_{0 k}(r)=C_{1} Y_{21}(r)+C_{2} Y_{22}(r) \\
& u_{0 k}(r)=C_{1} Y_{11}(r)+i C_{2} Y_{12}(r), v_{0 k}(r)=C_{1} Y_{21}(r)+i C_{2} Y_{22}(r)
\end{aligned}
$$

First formula here corresponds to the case $q>\beta$, second one to the case $\mathrm{q}<\beta$. The constants are the unknown real constants that will be found later.

The particular solution of the inhomogeneous equation is constructed with the using of the fundamental matrix. This fundanebtal matrix was constructed by the help of the method that is given in [16] in the form

$$
\mathrm{T}_{0}(r, \rho)=\int_{0}^{\infty} s \mathbf{H}_{0}(r, s) \mathbf{M}_{0 k}^{-1} \mathbf{H}_{0}(\rho, s) d s
$$

All elements of this matrix are expressed through the integrals of such form

$$
\int_{0}^{\infty} \frac{s^{m} J_{\mu}(r s) J_{v}(\rho s)}{\left(s^{2}+\mathrm{A}^{2}\right)\left(s^{2}+\mathrm{B}^{2}\right)} d s=T_{\mu, v}^{m}
$$

where $\mathrm{m}=01,2,3$,

$\mathrm{A}^{2}=\beta^{2}-q^{2}, \quad \mathrm{~B}^{2}=\frac{\left(q^{2}(1-2 v)+2 \beta^{2}(v-1)\right)}{2(1-v)}$. These integrals are reduced ti the known table integrals [15]. The particular solution of the inhomogeneous equation is constructed. The formulas for the displacements are obtained in the obvious form. The unknown constants, contained in the general solution will be defined by the satisfying the boundary functionals.

\section{The Transformation of the initial boundary Problem for the Case $n \geq 1, k \geq 1$}

Before the consideration of the general case of the Fourier's transformation parameters $\mathrm{n} \geq 1, \mathrm{k} \geq 1$, one must input the new unknown function associated with the original ones by the correspondences

$W_{n k}^{(1)}(r)=u_{n k}(r)+v_{n k}(r), W_{n k}^{(2)}(r)=u_{n k}(r)-v_{n k}(r), W_{n k}^{(3)}(r)=W_{n k}(r)$

A result we obtain in the transformations' domain the system of the movements' equations with regard of these new functions. This allows to construct the boundary value vector problem and apply the scheme, which was presented earlier, but for the matrixes of 3 by 3 dimension.

The application of the inverse Fourier's transformation completes the solution's construction

$$
\begin{aligned}
& \tilde{u}(r, \varphi, z)=\frac{2}{h} \sum_{k=1}^{\infty} u_{0 k}(r) \cos \beta_{k} z+\frac{4}{\omega h} \sum_{n=1}^{\infty} \sum_{k=0}^{\infty} u_{n k}(r) \cos \alpha_{n} \varphi \cos \beta_{k} z \\
& \tilde{w}(r, \varphi, z)=\frac{2}{\omega h} \sum_{k=1}^{\infty} w_{0 k}(r) \sin \beta_{k} z+\frac{4}{\omega h} \sum_{n=1}^{\infty} \sum_{k=0}^{\infty} w_{n k}(r) \cos \alpha_{n} \varphi \cos \beta_{k} z \\
& \tilde{v}(r, \varphi, z)=\frac{4}{\omega h} \sum_{n=1}^{\infty} \sum_{k=0}^{\infty} v_{n k}(r) c \cos \alpha_{n} \varphi \cos \beta_{k} z
\end{aligned}
$$

The obtained series are the conditionally convergent ones. That is connected with application of the integral transformation regard of the variable $\mathrm{z}$ by the inhomogeneous boundary conditions. So why, before the calculations of the stress values and the differentiation of the displacements' formulas, one need to extract the weak convergent part of the series. This was done and series are summarized with the known formulas [15].

\section{A. The Results of the Numerical Analyses}

The main goal of the numerical investigation in this problem is to identify the eigenfrequencies of the elastic wedge plate. They were investigated, and the dependence between their values and plate's geometrical parameters was established. The values of the eigenfrequencies are inversely proportional as to the relative radius of the plat, so and to the angle of the wedge plate's opening. A more complicated picture of the eigenfrequencies distribution is observed when an angle of the wedge is smaller than $\omega=\pi / 4$. With the increasing of the dimensionless radius on the segment $3 \leq R \leq 7$, the segment of the frequencies, at which eigenfrequecies appear, isn't changed. These segment lie in the frequency range $1,55 \leq \omega \leq 1,56$. As described in detail in [11], the resonance phenomenon corresponds to the such segments. Also it was established that with the increasing of Poisson's 
coefficient value, the range, in which lies the edge resonance frequency, is increased also. The significant effect on the frequency of the plate's edge resonance has the angle of the plate's opening. Also was admitted that the increasing of the relative radius leads to the increasing of the edge resonance's frequency too.

Also it was found at what frequency of the forced oscillations begins the separation of the basis on which the conditions of the ideal contact are given. To do this, the values of the static normal stress on the bottom face in the case of the wedge plate loading by its dead weight (at the edge $r=a$ are assumed the conditions of the ideal contact) were calculated. The results were compared with the values of the same normal dynamic stress that arise in a similar problem when uploading the upper face with the oscillating force through an absolutely rigid plate is providing. Calculations showed that by the values of the forced oscillations' frequency $\omega \leq 62$ the separation of the plate base's points with an opening angle $\omega=\pi / 3$ is not observed. A significant impact on the value of the frequency separation has the angle of the wedge opening. Thus, the angle of the plate $\omega=\pi / 6$ corresponds to the frequency $\omega=1,65$ and angle $\omega=\pi / 4$ - frequency $\omega=1,96$.

\section{Acknowledgment}

The research is supported by Ukrainian Department of Science and Education under Project N 0109U000920..

\section{References}

[1] A. Vatulyan, E. Chebakova, "The fundamental solutions for the orthtropic elastic medium in the case of the steady state oscillations", Prikl. Mechanica and Technich. Physica, vol.5, pp. 131-139, 2004.

[2] V. Grinchenko, The Equilibrium and steady-state Oscillations of the elastic Bodies. Naukova Dumka: Kiev, 1978.

[3] E. Starovoytov, V. Kubenko, and D. Tarlakovsky, "The oscillations of the round threelayered plates connected with the elastic base", Izv. Vuzov. Aiacionnaya technical, vol.2, pp. 16-29, 2009.

[4] CW. Bert, Research on dynamic Behavior of composite and sandwich Plates, part I, Shock Vibr. Digest, vol. 23(6), pp. 3-14, 1991.

[5] I. Vorovich, V. Babeshko, The dynamic mixed elasticity Problems for the nonclassic Areas, Nauka: Moscow, 1979.

[6] V. Mykhas'kiv, P. Martin and O. Kalynyak, "Time-domain BEM for 3$\mathrm{D}$ transient elastodynamic problems with interacting rigid movable discshaped inclusions", Computational Mechanics, vol. 53(6), pp. 1311$1325,2014$.

[7] V. Alexandrov, D. Pozharskii, "The problem of an inclusion in a three dimensional elastic wedge", Journal of Applied Mathematics and Mechanics, vol. 66(4), pp. 617-628, 2002.

[8] G. Popov, "The exact solution of the first main problem of elasticity for the thick wedge-like infinite elastic plate", Doklady RAN, vol. 381(6), pp. 782-785, 2001.

[9] D. Zhou, F.Au and Y. Cheng, "Three-dimensional vibration analyses of circular and annular plates via Chebyshev-Ritz method", Int. J. Solids Struct., vol.40 (12), pp. 3089-3105, 2003.

[10] A. Houmat, "Three-dimensional hierarchical finite element free vibration analysis of annular sector plates', J. Sound Vibr., vol. 276 (12), pp. 181-193, 2004.

[11] V. Grinchenko, V. Meleshko, The harmonic Oscillations and Waves in the elastic Bodies, Naukova Dumka: Kiev, 1981.

[12] R. Xu, "Three-dimensional exact solutions for the free vibration of laminated transversely isotropic circular, annular and sectorial plates with unusual boundary conditions", Arch. Appl. Mech., vol. 78, pp. 543$558,2008$.

[13] G. Popov, S. Abdymanapov and V. Efimov, Green's Functions and Matrices of the one-dimensional boundary Problems. Ruan: Almaty, 1999.

[14] R. Gantmakcher, Matrices' Theory. Gostexteorizdat: Moscow, 1954.

[15] I. Gradshteyn, I. Rygik, Tables of Integrals, Sums, Series and Productions. Fizmatliz: Moscow, 1963.

[16] N.Vaysfel'd, G. Popov and V. Reut, "The axisymmetric mixed problem of elasticity theory for a cone clamped along its side surface with attached spherical segment", J. Appl. Math. Mech., Vol. 77(1), pp. 7078, 2003.

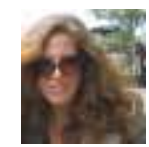

Doctor of Science, Professor, Head of the Mathematical Physics Department in Institute of Mathematics, Economics and Mechanics, Odessa Mechnikov University 